\title{
Molecular tracers of photo-evaporating disks around young stars
}

\author{
T. K. Nguyen, S. Viti, and D. A. Williams \\ Department of Physics and Astronomy, University College London, Gower Street, London, WC1E 6BT, UK
}

Received 5 November 2001 / Accepted 25 March 2002

\begin{abstract}
Disks around massive young stellar objects, and disks around low-mass stars irradiated by nearby OB associations, are eroded by photo-evaporation. In the latter case, this erosion may be an important factor in planet formation. As Johnstone et al. (1998) have shown, photo-evaporating material is gravitationally retained within a critical radius from the star, and constitutes an envelope similar to a Photon-Dominated Region (PDR) that normally arises at the edge of a molecular cloud irradiated by a massive star. We explore the chemistry of such a PDR/disk system to examine the contribution that it may make to the molecular species that may be observed. The model is in two phases; firstly, a collapse from low density to a high density appropriate for a disk; and, secondly, a 2D calculation of the irradiation of disk material by the radiation field of the central massive star or nearby OB association. The model follows the chemistry self-consistently through both phases. We compute the column densities of species through the PDR/disk system, averaged over the disk. We validate our model by comparing predicted averaged molecular column densities with those of several species detected in the disk around the 10 solar mass star GL 2591, currently the sole example known of this kind of object. Results are in good agreement for a model in which the outer part of the PDR is hot while the inner part is cool, and in which the local ionization rate is comparable with that caused by cosmic rays in the local interstellar medium. We show that in addition to the four detected species, there should be many others also detectable in this system, including $\mathrm{HCN}, \mathrm{NH}_{3}$ and CS. Similar conclusions should apply to other disks around massive stars. Disks around low-mass stars are much more common; our models show that when irradiated by a nearby OB association such disks with their attendant PDRs also generate a rich chemistry. No detections of molecules in such objects have yet been reported. However, the models suggest that averaged molecular column densities should be comparable to those detected in disks around massive stars (see references listed in Table 4 for molecules in GL 2591). Potential tracers of irradiated disks around low-mass stars include $\mathrm{OH}, \mathrm{CH}_{3}$ and $\mathrm{C}_{2} \mathrm{H}$. We note that the detection in a disk of PDR-type chemistry is a clear signature that the disk is undergoing erosion. Its duration is therefore limited, with consequences for planet formation.
\end{abstract}

Key words. IMS: clouds - ISM: molecules - circumstellar matter

\section{Introduction}

Circumstellar disks around young stars, the material of future planetary systems, have been the subject of much research in recent years. However, observations of these disks are not easy due to confusion with foreground and background gas and their complex geometry. Nevertheless, the Hubble Space Telescope (HST) has provided strong dynamical evidence of the existence of extended circumstellar disks around many low-mass young stars (e.g. Bally et al. 1998), and there is also evidence that disks are present in OB associations (O'Dell 1998, and references therein) as well as around massive stars (Mitchell et al. 1989; Carr et al. 1995).

Radio and IR observations of molecular abundances in circumstellar disks can reveal the chemical connection between planetary and interstellar matter. Furthermore, the sizes of disks are still not well determined: the knowledge

Send offprint requests to: T. K. Nguyen,

e-mail: tkn@star.ucl.ac.uk of the molecular abundances at each radius of a disk may be helpful in determining its geometry. Dutrey et al. (1997) report molecular emissions from disks around the low mass T Tauri stars DM Tau and GG Tau and infer that the disk may extend to 800-1000 AU. They also find diversity in the chemistry: some of the detected species are under-abundant with respect to dark cloud abundances by factors of 5 to 30 , indicating that depletion on to grains is occurring, while large ratios of $\mathrm{C}_{2} \mathrm{H} / \mathrm{HCO}^{+}$and $\mathrm{CN} / \mathrm{HCN}$ are indicative of photo-dominated chemistry.

It is likely that disks are flared, allowing them to absorb more of the radiation from the central star at large radii than possible for a flat disk. This would result in the formation of a layer of warm gas and dust at the surface of the disk (Chiang \& Goldreich 1997). Goldsmith et al. (1999) also note that some emission must occur from the surface layers of flared disks: from observations of methanol, they infer that gas species are desorbed from the grains in these layers while retained on the icy mantles in the midplane region of the disk. Such observations 
indicate that substantial chemical processing, desorption and depletion, occurs in the evolution of disks surrounding low mass stars.

Extended radio emission has been observed in the Trapezium region of the Orion OB association. This emission is thought to be produced by photo-evaporation of the surface of disks surrounding low-mass stars (Johnstone et al. 1998). Photo-evaporation via ultraviolet photons could be a very efficient mechanism for the removal of disks and an important factor in any subsequent planetary formation. Given a strong enough UV field, the disk surface can be heated to temperatures in excess of $1000 \mathrm{~K}$, and over most of the disk the gas material becomes gravitationally unbound. Even if the central star is of low mass a disk may be exposed to a strong UV radiation field from an external nearby massive star. External radiation from an $\mathrm{OB}$ association in the vicinity of the disk would evaporate circumstellar disks much faster than the radiation field of the central low-mass star, consistent with the timescales required for solar-type systems (Johnstone 2000). However, disk material sufficiently close to the central star in either case will remain gravitationally bound to the system. The radius at which the heated material is no longer bound by gravity is called the critical gravitational radius, $r_{\mathrm{g}}$. Within this radius the evaporated but gravitationally bound material creates an extended photondominated zone, i.e. a PDR. The potential chemical role of this PDR is the subject of the present paper.

Although disks surrounding low-mass stars are more common, there is evidence that disks can also survive around young massive stars. Mitchell et al. (1989) reported detection of circumstellar $\mathrm{CO}$ from the infrared source GL 2591, a $10 M_{\odot}$ young stellar object (YSO) (van der Tak et al. 1999). This object is thought to have an optically thick dust shell. The CO detected by Mitchell et al. (1989) shows a variety of structures and gas properties, and in particular they deduce the presence of a hot gas $(T \sim 1000 \mathrm{~K})$ at the surface of the disk. Carr et al. (1995) extended the study of GL 2591 to other species. Their analysis indicates that most of the emission arises in gas at $\sim 200$ and $1000 \mathrm{~K}$, corresponding to the components observed by Mitchell et al. (1989). They propose a scenario where grains play a passive role. During the star formation process, molecules freeze on to grains and they are then released after the star is formed, by direct heating of the dust by the star, by photo-desorption or other mechanism.

From this limited, yet convincing, set of observations, it is clear that disks may extend up to $\sim 1000 \mathrm{AU}$, still moving in Keplerian motion around the central star and that molecular emission may arise from different components of the disk.

Detailed chemical models are required to help interpret these results. Although the majority of the early models dealt only with the midplane region (Aikawa et al. 1997; Aikawa et al. 1998), recent models by Aikawa \& Herbst (1999) and Willacy \& Langer (2000) have taken into consideration the chemical distribution in all layers of the disk from the midplane to the surface layers. Willacy \& Langer (2000) find that the UV radiation from the central source is important in driving the chemistry. They also infer that the observed emission comes from a layer below the surface where UV photons can still penetrate sufficiently to drive desorption but molecules are shielded to a certain extent from photo-dissociation. However, their models do not incorporate an extended PDR within the critical gravitational radius created from evaporated yet gravitationally bound gas. Two-dimensional models of a low stellar mass system have also been made by van Zadelhoff et al. (2001). The authors modelled their molecular line emissions in order to derive the density and temperature structures of the disk.

In this paper we investigate a model in which a circumstellar disk is being heated by a strong radiation field from a massive star that may be the central object in the disk, or nearby. Within the critical radius, $r_{\mathrm{g}}$, the heated gas is trapped at the surface of the disk (Johnstone 2000), in effect creating a photon dominated region (a PDR), while outside this region the disk is being photoevaporated. PDRs have a characteristic chemistry caused by the influence of the radiation field on the dense and warm molecular gas. We shall explore the chemistry in a PDR associated with a circumstellar disk undergoing photo-evaporation. Our intention is to identify molecular tracers of disks in this destructive phase of their evolution, from the gas that is trapped in the PDR. The model predictions will be compared with the rather limited observational data for GL 2591, a candidate high mass object for a photo-evaporating object that should include a PDR. Predictions of potentially observable species, as yet undetected, arising in the eroding $\mathrm{PDR} /$ disk system are also made.

Disks around low-mass stars are much more numerous than those associated with high mass stars. Such disks will incorporate a PDR when irradiated by a nearby massive star. In such a case, the PDR may provide molecular signatures of the late evolutionary stage of a disk around a low-mass object. Therefore, we shall also consider PDRs arising in disks around a low-mass star, where the radiation source is assumed to be a nearby massive star. Such systems have been proposed by Johnstone et al. (1998), and in this paper we show that potential molecular tracers can be identified.

The model is described in Sect. 2. A comparison with observational results for GL 2591 is given in Sect. 3, and some predictions of other species potentially detectable in disks around massive stars are given in Sect. 4. Predictions of molecular tracers of eroding disks around low mass stars are given in Sect. 5. A brief conclusion is presented in Sect. 6.

\section{The model}

The model calculation is carried out in two phases. In the first phase, initial abundances for the second phase are computed, see Sect. 2.1. The second phase describes 
the time-dependent two-dimensional behaviour of the disk and PDR system, see Sect. 2.2.

\subsection{The calculation of initial abundances}

In this phase, we consider a single-point collapse for a cloud forming a star-disk system. The initial and final number densities adopted in this phase are $10^{3}$ and $10^{7} \mathrm{~cm}$ and the collapse is assumed to be free-fall. This calculation is required in order to derive the initial atomic and molecular abundances in the disk. Since this first phase is a single-point calculation, the initial abundances for the second phase disk calculation are the same for all $r$ and $z$. As noted above, the second phase calculation is then a time-dependent two-dimensional calculation. The collapsing model takes into account freeze-out and surface hydrogenation (as in Viti \& Williams 1999a). The initial elemental abundances for our collapsing cloud are given in Table 2. The chemistry includes 337 species in a network of 2950 chemical reactions taken from the UMIST database (Millar et al. 1997). Although the highest densities in the disk midplane are on the order of $10^{10} \mathrm{~cm}$, three body reactions have not been included. Our interest in this work focuses on the potential contribution to the chemistry from the PDR in which the densities are somewhat lower.

We also made an alternative calculation of initial molecular abundances for the second phase. In the first phase of our calculations, instead of assuming that at late times the gas-phase species accreted onto grain surfaces, which subsequently underwent hydrogenation reactions, we suppressed freeze-out so that all species remained in the gas-phase. This purely gas-phase description was then used as the initial composition of the disk at the start of Phase 2.

We stop the collapse phase of the model when the gas number density reaches its final specified value of $10^{7} \mathrm{~cm}$.

\subsection{The chemical evolution of the disk and PDR system}

Our model follows the time-dependent chemical evolution of the envelope of a disk at different radii $(r)$ from the central object out to a critical radius, $r_{\mathrm{g}}$. Beyond $r_{\mathrm{g}}$, the envelope is no longer gravitationally bound, and we do not consider the chemistry in that zone. Our model is two dimensional in that we compute the time dependent chemical evolution as a function of $r$ and of $z$, the distance from the central plane of the disk. The driving source of the PDR envelope is either from the central star, in the case of a massive YSO, or from an external massive star or stars, as in the case of disks surrounding low mass stars in OB association, such as the Trapezium in Orion.

The radiation field from a central massive star is taken to be $4 \times 10^{5} G_{0}$ at $r=20$ AU (where $G_{0}$ represents the mean interstellar radiation field intensity) falling off as $\left(r^{2}+z^{2}\right)^{-1}$, out to $r=r_{\mathrm{g}}$, where

$r_{\mathrm{g}}=G M_{*} \frac{\mu m_{\mathrm{H}}}{k T}$.

The interstellar radiation field is taken to be ambient and is therefore negligible.

This strong UV field will determine the temperature of the gas at each point in the envelope. The gas in the upper layer of the PDR is assumed to be heated to a temperature of $1000 \mathrm{~K}$ (e.g. Carr et al. 1995), while subsequent lower layers, due to shielding and cooling effects, are assumed to be at a temperature corresponding to the disk temperature at that given radius. The temperature of the midplane of the disk is calculated using Eq. (2) (Aikawa et al. 1997),

$T(r)=28\left(\frac{r}{100 \mathrm{AU}}\right)^{-1 / 2}\left(\frac{L_{*}}{L_{\odot}}\right)^{1 / 4} \mathrm{~K}$

where $L_{\star}\left(\right.$ GL 2591) $=9 \times 10^{4} L_{\odot}($ Lada et al. 1984), and the scale height, $H(r)$ is calculated from Eq. (3) (Hollenbach et al. 1994)

$H(r)=r_{\mathrm{g}}\left(\frac{r}{r_{\mathrm{g}}}\right)^{3 / 2}$

The disk is taken to be bounded at one scale height from the midplane at each radius, and the values of the scale height at various radii are given in Table 1.

The values for the physical and chemical parameters employed at various radii are summarized in Table 1 and the dependence of number density on $z$ is assumed to be given by Eq. (4),

$n(r, z)=n_{\mathrm{o}}(r) \exp \left(\frac{-z^{2}}{H(r)^{2}}\right)$

The PDR model follows the time-dependent evolution of the same chemistry as in the first phase, using the output of that phase as the initial abundances, and the evolution is followed as a function of both $r$ and $z$. CO self-shielding is allowed for in a manner similar to that described by Wagenblast (1992), based on the detailed study of van Dishoeck \& Black (1988). However, it should be noted that the temperature and density structure in the PDR in this calculation is imposed, rather than being self-consistently determined (which would be computationally expensive to include).

YSOs are considered to be strong sources of X-rays which may significantly affect the chemistry of the disk. Igea \& Glassgold (1999) predict that stellar X-rays can penetrate the entire disk. In some models, therefore, we simulate the presence of X-ray ionization by enhancing our standard cosmic ionization rate, $1 \zeta\left(1 \zeta=1.3 \times 10^{-17} \mathrm{~s}^{-1}\right)$ up to $10^{5} \zeta$.

The UV radiation field from the central object (or from an external OB source) is then assumed to be switched on, and irradiates the two-dimensional disk. The chemical evolution of the envelope of the disk is followed at 12 different radii (as specified in Table 1 ). At each radius 
Table 1. Parameters for each radial point studied for a $10 M_{\odot}$ star. The notation $a(b)$ implies $a \times 10^{b}$.

\begin{tabular}{cccc}
\hline \hline Radius $(\mathrm{AU})$ & $n(r, z=0)\left(\mathrm{cm}^{-3}\right)$ & $T(r)$ at midplane $(\mathrm{K})$ & $H(r)(\mathrm{AU})$ \\
\hline 40 & $2.59(10)$ & 767 & 6.86 \\
60 & $8.51(09)$ & 626 & 12.60 \\
80 & $3.86(09)$ & 542 & 19.40 \\
100 & $2.09(09)$ & 485 & 27.12 \\
200 & $3.10(08)$ & 343 & 76.70 \\
300 & $1.02(08)$ & 280 & 140.90 \\
400 & $4.61(07)$ & 242 & 216.93 \\
600 & $1.51(07)$ & 198 & 398.53 \\
800 & $6.85(06)$ & 171 & 613.57 \\
1000 & $3.71(06)$ & 153 & 857.49 \\
1200 & $2.24(06)$ & 140 & 1127.20 \\
1360 & $1.59(06)$ & 132 & 1360.00 \\
\hline
\end{tabular}

Table 2. The elemental abundances of initial atoms relative to that of hydrogen nuclei. Note $a(b) \equiv a \times 10^{b}$ (see Ruffle et al. 1998).

\begin{tabular}{cc}
\hline \hline Species & Elemental Abundance \\
\hline $\mathrm{H}$ & $0.0(-00)$ \\
$\mathrm{He}$ & $7.1(-02)$ \\
$\mathrm{C}$ & $1.0(-04)$ \\
$\mathrm{N}$ & $2.0(-05)$ \\
$\mathrm{O}$ & $2.0(-04)$ \\
$\mathrm{S}$ & $3.0(-08)$ \\
$\mathrm{Mg}$ & $3.0(-09)$ \\
$\mathrm{Na}$ & $1.0(-06)$ \\
$\mathrm{Si}$ & $8.0(-09)$ \\
\hline
\end{tabular}

the envelope, treated as a PDR, is represented by a slab subdivided into five layers represented by 5 depth points. The temperature at the edge of the disk (i.e. furthest from the midplane of the disk) is taken to be $1000 \mathrm{~K}$, and the temperatures of inner layers of the PDR are defined for the models listed in Table 3.

When the UV field is switched on, the icy mantles are assumed to be instantaneously evaporated. For each radius, we have computed a grid of models (see Table 3 ) in which we vary the ionization rate and temperature within the envelope. This is done in order to investigate the effect of the X-ray ionization on the disk. The chemical composition of the PDR was then calculated for each model.

\section{Results and discussion}

The results for Models A-G are given in Table 4. The table contains the averaged weighted column densities over the entire area of the disk for $r<r_{\mathrm{g}}$. The disk is assumed to be face-on, and entirely within the telescope beam. The column densities shown in all cases are calculated from the edge to the disk midplane. Table 4 also compares the model results with all known molecular observations of GL 2591, the best candidate for the high stellar mass scenario being studied here. As stated in Sect. 1, GL 2591 has been observed by Carr et al. (1995) to have hot gas at the surface of the disk. Five separate observations have been made to date by Mitchell et al. (1989); Carr et al. 1995; Helmich et al. (1996); Lahuis \& van Dishoeck (2000) and Boonman et al. (2000).

We found that the models where freeze-out is suppressed produced column densities in the PDR of the disk lower than observed. These models are not shown. This mainly affected species such as $\mathrm{NH}_{3}, \mathrm{CH}_{4}, \mathrm{HCN}$ and $\mathrm{H}_{2} \mathrm{O}$ which are further produced in the collapse phase via hydrogenation reactions of the grain surfaces. Therefore, we chose to use the models in which freeze-out occurs in Phase 1 , followed by evaporation in the second phase. This scenario has also been proposed by Carr et al. (1995) who show that their observations can be explained by models in which gas-phase species are preserved on grain mantles and later released at high temperatures.

We discuss first the sensitivity of the model to the parameter changes representing the ionization rate and the assumed temperature of the inner layers of the PDR. Models A, B and C represent the effect of changing the ionization rate by factors of $1,10^{3}$, and $10^{5}$ respectively for a given temperature structure. It has previously been shown (Lepp \& Dalgarno 1996) that the presence of Xrays causes an enhancement in certain species, including $\mathrm{NO}, \mathrm{CN}, \mathrm{HCN}$ and $\mathrm{CH}$, although this enhancement in abundance is not seen in all species. Molecules such as CO and $\mathrm{N}_{2}$ are more readily dissociated due to the enhanced ionization rate, and this dissociation produces free atoms, which are then used in the formation of simple molecules such as $\mathrm{HCN}$ and $\mathrm{NH}_{3}$. For models $\mathrm{A}, \mathrm{B}$ and $\mathrm{C}$, changes in our computed column densities arising from changes in the ionization rate are always by less than a factor of five, and often less than a factor of two. Evidently, as the ionization rate changes, at the high number densities of the gas considered here the formation and destruction 
Table 3. The Grid Model parameters for each radial point. The notation $a(b)$ implies $a \times 10^{b}$ and abbreviation PDRE $-\mathrm{Photon}$ Dominated Region Envelope.

\begin{tabular}{cccc}
\hline \hline Model & Scenario & Temp. of Lower Layers $(\mathrm{K})$ & Mass of Central Star \\
\hline A & PDRE with $1 \zeta$ & midplane value & $10 M_{\odot}$ \\
B & PDRE with $1(03) \zeta$ & midplane value & $10 M_{\odot}$ \\
C & PDRE with $1(05) \zeta$ & midplane value & $10 M_{\odot}$ \\
D & PDRE with $1 \zeta$ & $1000 \mathrm{~K}$ & $10 M_{\odot}$ \\
E & PDRE with $1(03) \zeta$ & $1000 \mathrm{~K}$ & $10 M_{\odot}$ \\
F & PDRE with $1(05) \zeta$ & $1000 \mathrm{~K}$ & $10 M_{\odot}$ \\
G & No PDRE present and $1 \zeta$ & midplane value & $10 M_{\odot}$ \\
& & & \\
H & PDRE with $1 \zeta$ & midplane value & $1 M_{\odot}$ \\
I & PDRE with $1 \zeta$ & $100 \mathrm{~K}$ & $1 M_{\odot}$ \\
J & No PDRE present and $1 \zeta$ & midplane value & $1 M_{\odot}$ \\
\hline
\end{tabular}

processes are more or less equally affected. This conclusion is also evident in the results from models $\mathrm{D}, \mathrm{E}$ and $\mathrm{F}$, which also show the effect of changing the ionization rate in a PDR with a different temperature structure.

The effects of temperature are more pronounced. We may compare the pairs $\mathrm{A}$ and $\mathrm{D}, \mathrm{B}$ and $\mathrm{E}$, and $\mathrm{C}$ and $\mathrm{F}$, each pair having the same ionization rate. In models $\mathrm{D}$, $\mathrm{E}$ and $\mathrm{F}$ (which are probably less realistic than models $\mathrm{A}$, $\mathrm{B}$ and $\mathrm{C}$ ), the higher temperature layers are assumed to penetrate deeper than in models A, B and C. Apart from the case of $\mathrm{CO}$, the models with higher temperatures at greater depths show greater column densities, in several cases - notably $\mathrm{NH}_{3}$ - by more than one order of magnitude. This result simply reflects the effect of temperature increases on mildly endothermic reactions included in the chemical network. The increased abundances of $\mathrm{HCN}$, $\mathrm{CH}_{4}$ and $\mathrm{NH}_{3}$ can be explained by considering their main formation routes: $\mathrm{H}_{2}+\mathrm{CN}, \mathrm{H}_{2}+\mathrm{CH}_{3}$ and $\mathrm{H}_{2}+\mathrm{NH}_{2}$ for the formation of $\mathrm{HCN}, \mathrm{CH}_{4}$ and $\mathrm{NH}_{3}$ respectively. These reactions have energy barriers, therefore making them more significant in the high temperature regimes studied here. $\mathrm{C}_{2} \mathrm{H}_{2}$ also shows a sharp increase in abundance which is also due to the increased temperatures. At high temperatures, oxygen atoms become locked into $\mathrm{H}_{2} \mathrm{O}$ and it is this increase in water abundance which produces the increase in $\mathrm{C}_{2} \mathrm{H}_{2}$ column densities via the reaction $\mathrm{C}_{2} \mathrm{H}_{3}^{+}+\mathrm{H}_{2} \mathrm{O}$. No significant increase in abundance is seen for either CS or $\mathrm{SiO}$. This is because neither their main formation or destruction mechanisms have energy barriers and therefore are unaffected by the increase in temperature.

The chemical effect of including the PDR may be estimated by comparing results of Model $\mathrm{G}$ with those of Models A-F. Model G conditions consisted of only a cold, dense disk, without the corresponding PDR envelope above the surface of the inner disk. For consistency, in this case $(\mathrm{G})$ we have assumed only an ambient interstellar radiation field, and therefore the temperatures of the disk remain relatively low. The results are given in Table 4 . Since Model G includes only the disk, the molecular column densities achieved in this model are significantly less than those in other models describing the disk and PDR system. The PDR clearly contributes very significantly to the predicted column densities of all the species listed, most notably so in the case of $\mathrm{C}_{2} \mathrm{H}_{2}$ which is increased in the case of models A-C by about two orders of magnitude and for models D-F by over three orders.

It is of interest to compare the model results with the column densities inferred from observations. It should, however be noted that the models have not been designed to "fit" any particular object, rather to illustrate the contribution of a PDR and its sensitivity to ionization rate and temperature structure. We do not, therefore, expect to find a perfect match. Nevertheless, the results are very encouraging for the small list of molecules for which detections or upper limits are available. In general, model A provides reasonable agreement, apart from the case of $\mathrm{C}_{2} \mathrm{H}_{2}$. As we have seen, the temperature sensitivity of this species is much greater than that of other species, so a modest increase in a PDR temperature distribution in the envelope should provide enough $\mathrm{C}_{2} \mathrm{H}_{2}$ to give a reasonable match. This is in contrast to the results of Millar et al. (1991) and Charnley \& Tielens (1992) who argued that a hot gas-phase chemistry was unable to produce observed quantities of $\mathrm{C}_{2} \mathrm{H}_{2}$. It has since been proposed that if sufficient abundances of $\mathrm{C}_{2} \mathrm{H}_{2}$ are to be produced, the molecule must be present in ice mantles on grain surfaces, and an abundance of $0.1-0.5 \%$ of water ice is needed (Lahuis \& van Dishoeck 2000). The subsequent sublimation of the ice is predicted to produce the high abundances observed. Although there are no grain surface reactions in the rate file used in this paper, a number of reactions to generate $\mathrm{C}_{2} \mathrm{H}_{2}$ are known, including Reactions 5-8 which have been taken from the Ohio rate file (Ruffle \& Herbst 2000).

$$
\begin{aligned}
& \mathrm{GH}_{2}+\mathrm{GC}_{2} \mathrm{H} \longrightarrow \mathrm{GC}_{2} \mathrm{H}_{2} \\
& \mathrm{GCH}_{2} \mathrm{CO} \longrightarrow \mathrm{GC}_{2} \mathrm{H}_{2}+\mathrm{GO} \\
& \mathrm{GC}_{2} \mathrm{H}_{4} \longrightarrow \mathrm{GC}_{2} \mathrm{H}_{2}+\mathrm{GH}_{2} \\
& \mathrm{GH}_{2} \mathrm{C}_{3} \mathrm{~N} \longrightarrow \mathrm{GC}_{2} \mathrm{H}_{2}+\mathrm{GCN}
\end{aligned}
$$


Table 4. Comparison of Observed Column Densities of species towards GL 2591 with Weighted Averaged Column Densities over the entire disk, for Models A-G for the same selection of species. Observations taken from: ${ }^{i}$ Mitchell et al. (1989), ${ }^{j}$ Carr et al. (1995), ${ }^{k}$ Lahuis \& van Dishoeck (2000), ${ }^{m}$ Boonman et al. (2000), ${ }^{n}$ Helmich et al. (1996). Notation $a(b) \equiv a \times 10^{b}$.

\begin{tabular}{ccccccccc}
\hline \hline \multicolumn{7}{c}{ Column Densities $\left(\mathrm{cm}^{-2}\right)$ for Models } \\
\hline Species & Observed $\left(10^{16}\right)$ & $\mathrm{A}$ & $\mathrm{B}$ & $\mathrm{C}$ & $\mathrm{D}$ & $\mathrm{E}$ & $\mathrm{F}$ & $\mathrm{G}$ \\
\hline $\mathrm{CO}^{i}$ & $560 \pm 110$ & $4.14(18)$ & $4.10(18)$ & $3.03(18)$ & $6.54(17)$ & $4.80(17)$ & $4.77(17)$ & $2.21(17)$ \\
$\mathrm{HCN}^{j}$ & $1.6 \pm 0.5$ & & & & & & & \\
$\mathrm{HCN}^{k}$ & $4.0 \pm 0.6$ & $6.64(16)$ & $7.13(16)$ & $7.18(16)$ & $2.18(17)$ & $2.81(17)$ & $4.79(17)$ & $1.86(16)$ \\
$\mathrm{HCN}^{m}$ & 6.6 & & & & & & & \\
$\mathrm{C}_{2} \mathrm{H}_{2}{ }^{j}$ & $1.05 \pm 0.16$ & $2.74(14)$ & $3.44(14)$ & $2.91(14)$ & $1.17(16)$ & $0.51(16)$ & $0.48(16)$ & $4.29(12)$ \\
$\mathrm{CH}_{4}^{j}$ & $<130$ & $1.44(17)$ & $2.88(16)$ & $8.51(16)$ & $2.57(18)$ & $1.43(18)$ & $1.25(18)$ & $6.40(16)$ \\
$\mathrm{NH}_{3}^{j}$ & $<0.7$ & $3.91(16)$ & $1.66(16)$ & $1.66(16)$ & $1.59(17)$ & $3.97(17)$ & $1.18(17)$ & $6.93(14)$ \\
$\mathrm{CS}^{j}$ & $<0.9$ & $1.1(14)$ & $2.78(14)$ & $2.16(14)$ & $2.67(14)$ & $7.40(14)$ & $7.84(14)$ & $2.36(13)$ \\
$\mathrm{SiO}^{j}$ & $<2.5$ & $4.56(14)$ & $4.30(14)$ & $1.73(14)$ & $3.16(14)$ & $7.25(12)$ & $2.36(12)$ & $2.43(13)$ \\
$\mathrm{H}_{2} \mathrm{O}^{n}$ & 200 & $2.22(18)$ & $1.90(18)$ & $1.55(18)$ & $8.50(18)$ & $8.67(18)$ & $8.62(18)$ & $3.20(17)$ \\
\hline
\end{tabular}

where $\mathrm{GX}_{i}$ represents species $\mathrm{X}_{i}$ on a grain surface. The surface formation of $\mathrm{C}_{2} \mathrm{H}_{2}$ may be especially efficient for reactions 5 and 6 as the grain surface fractional abundance of $\mathrm{C}_{2} \mathrm{H}$ and $\mathrm{CH}_{2} \mathrm{CO}$ are sufficiently high $\left(\approx 10^{-7}\right.$ and $10^{-8}$ respectively).

\section{Other potentially detectable molecules in disks around massive stars}

So far, only four molecules (see Table 4) have been detected in disks around isolated massive stars, while upper limits have been estimated for a further four species. In this section we investigate the chemistry of some other species previously observed in other regions, and predicted by our models to be also abundant in disks around massive stars. Table 5 lists these species for models A, B and C. We list only those species that are sufficiently abundant to be detectable and which have been observed elsewhere in the interstellar medium and/or in comets.

Comparing the predicted abundances shown in Table 5 with those found in dark clouds (e.g. TMC-1), we find that, in general, many species, including $\mathrm{H}_{2} \mathrm{CS}, \mathrm{NH}_{3}$, $\mathrm{CH}_{2} \mathrm{CN}$, and $\mathrm{HC}_{3} \mathrm{~N}$, should be enhanced in disks around massive stars. We also notice that some species vary in fractional abundance with the ionization rate; examples are $\mathrm{CH}, \mathrm{C}_{2} \mathrm{H}$, and $\mathrm{HCO}^{+}$. By investigating and understanding the above behaviours, we can divide species into "classes" which may allow us to use different molecules as tracers of different properties of the disks. The case of $\mathrm{HCO}^{+}$is discussed below. Enhanced abundances with respect to dark clouds for some of the species, such as $\mathrm{NH}_{3}$ and $\mathrm{H}_{2} \mathrm{CS}$, may be easily explained: our initial abundances (as derived from Phase 1 of our calculations) are the result of the collapse of a cloud with subsequent freeze-out and hydrogenation on to grains. Once the UV radiation field is "switched on" (Phase 2), the mantle evaporates, injecting in the gas a high abundance of hydrogenated species such as $\mathrm{NH}_{3}$ and $\mathrm{H}_{2} \mathrm{CS}$. By detecting these species we may be able to determine the degree of depletion and hydrogenation in disk environments.

The abundance of $\mathrm{HCO}^{+}$increases by about 2 orders of magnitude when the ionization rate is increased by a factor of $10^{5}$. The main route of formation of $\mathrm{HCO}^{+}$is:

$$
\mathrm{H}_{3}^{+}+\mathrm{CO} \rightarrow \mathrm{HCO}^{+}+\mathrm{H}_{2} \text {. }
$$

Moreover, its abundance is in any case enhanced with respect to the observed abundances in dark or dense clouds (Liszt \& Lucas 2000; Scappini et al. 2000). This situation resembles the one found in Herbig-Haro objects (e.g. Girart et al. 1994) where an enhancement of $\mathrm{HCO}^{+}$is indirectly due to the high UV radiation field generated in the shock (Viti \& Williams 1999b). This enhancement arises from the rapid creation of $\mathrm{C}^{+}$by photo-processing of $\mathrm{CO}$ and the reaction of $\mathrm{C}^{+}$with abundant $\mathrm{H}_{2} \mathrm{O}$ released from grains. A similar process may be occurring in circumstellar disks. The UV radiation field is much higher than the one assumed to be present in the $\mathrm{HH}$ clumps; this is consistent with the larger abundance of $\mathrm{HCO}^{+}$. In fact, $\mathrm{HCO}^{+}$is potentially a "multiple" tracer. Firstly, it may trace high radiation field environments where depletion, hydrogenation and subsequent evaporation have occurred. Secondly it can be used to determine the level of X-ray ionization coming from the central star; and thirdly it can be a tracer of the disk evolution. The column densities in Tables 4-7 illustrating these points are shown at $\sim 10^{4}$ years after the disk envelope is formed. However, $\mathrm{HCO}^{+}$abundance is much higher at earlier stages of the disk evolution, due to the higher abundance of $\mathrm{H}_{3}^{+}$. An accurate determination of $\mathrm{HCO}^{+}$abundance could, therefore provide clues to the disk evolutionary stage.

\section{Disks around low-mass stars}

So far in this paper, we have only considered an isolated system of a disk around a massive star. A PDR envelope above a disk around a low mass star may also form by 
Table 5. Predicted Column Densities of Species for high stellar mass case, which may be observable. The cut-off column density is $10^{12} \mathrm{~cm}^{-2}{ }^{1}$ TMC-1 column densities were taken from Ohishi et al. (1992). Notation $a(b) \equiv a \times 10^{b}$.

\begin{tabular}{|c|c|c|c|c|}
\hline \multirow[b]{2}{*}{ Species } & \multicolumn{4}{|c|}{ Column Densities $\left(\mathrm{cm}^{-2}\right)$} \\
\hline & Model A & Model B & Model C & $\mathrm{TMC} 1^{1}$ \\
\hline $\mathrm{OH}$ & $5.92(16)$ & $6.19(16)$ & $9.89(16)$ & $3.00(15)$ \\
\hline $\mathrm{CH}$ & $1.27(12)$ & $2.04(13)$ & $1.57(15)$ & $2.00(14)$ \\
\hline $\mathrm{CH}_{3}$ & $1.06(14)$ & $2.82(14)$ & $4.85(15)$ & - \\
\hline $\mathrm{C}_{2}$ & $4.04(14)$ & $3.25(15)$ & $7.01(15)$ & - \\
\hline $\mathrm{C}_{2} \mathrm{H}$ & $1.19(12)$ & $2.39(14)$ & $9.04(14)$ & $5-10(14)$ \\
\hline $\mathrm{HCO}$ & $1.37(12)$ & $5.57(12)$ & $2.10(12)$ & - \\
\hline $\mathrm{HCO}^{+}$ & $2.79(13)$ & $2.70(14)$ & $3.34(15)$ & $8.00(13)$ \\
\hline $\mathrm{H}_{2} \mathrm{CO}$ & $2.65(14)$ & $1.35(14)$ & $1.54(14)$ & $2.00(14)$ \\
\hline $\mathrm{H}_{2} \mathrm{~S}$ & $7.72(13)$ & 1.64(13) & $5.99(12)$ & $<5.00(12)$ \\
\hline OCS & $1.96(14)$ & $3.47(13)$ & - & $2.00(13)$ \\
\hline CS & $1.10(14)$ & $2.78(14)$ & $2.16(14)$ & $1.00(14)$ \\
\hline HCS & $3.80(13)$ & $5.83(13)$ & $4.60(13)$ & - \\
\hline $\mathrm{H}_{2} \mathrm{CS}$ & $1.68(14)$ & $2.54(13)$ & $2.03(13)$ & $3.00(13)$ \\
\hline $\mathrm{SO}$ & $3.61(14)$ & $4.48(14)$ & $6.22(13)$ & $5.00(13)$ \\
\hline $\mathrm{SO}_{2}$ & $6.83(14)$ & $2.17(14)$ & $1.82(13)$ & $<1.00(13)$ \\
\hline $\mathrm{NH}$ & $1.97(13)$ & $3.35(13)$ & $1.23(14)$ & - \\
\hline $\mathrm{NH}_{2}$ & $1.06(13)$ & $4.22(13)$ & $2.27(14)$ & - \\
\hline $\mathrm{NH}_{3}$ & $6.49(15)$ & $3.91(16)$ & $1.66(16)$ & $2.00(14)$ \\
\hline $\mathrm{CN}$ & $1.24(12)$ & $3.25(13)$ & $7.47(14)$ & $3.00(14)$ \\
\hline $\mathrm{H}_{2} \mathrm{CN}$ & $1.53(12)$ & - & $1.05(12)$ & - \\
\hline $\mathrm{CH}_{2} \mathrm{CN}$ & $2.30(16)$ & $1.81(16)$ & $2.12(16)$ & $5.00(13)$ \\
\hline $\mathrm{HC}_{3} \mathrm{~N}$ & $5.66(15)$ & $1.29(16)$ & $3.03(15)$ & $6.00(13)$ \\
\hline $\mathrm{C}_{2} \mathrm{H}_{3}$ & $1.21(12)$ & - & $1.52(12)$ & - \\
\hline $\mathrm{C}_{2} \mathrm{H}_{4}$ & $1.57(14)$ & $1.15(13)$ & $5.14(13)$ & - \\
\hline $\mathrm{C}_{3}$ & $1.13(14)$ & $2.09(14)$ & $1.22(15)$ & - \\
\hline $\mathrm{HNC}$ & $3.12(16)$ & $2.67(16)$ & $2.16(16)$ & $2.00(14)$ \\
\hline $\mathrm{HC}_{5} \mathrm{~N}$ & $2.01(15)$ & $5.63(14)$ & $2.97(14)$ & $3.00(13)$ \\
\hline $\mathrm{C}_{3} \mathrm{H}_{4}$ & $3.69(12)$ & $2.91(12)$ & $5.52(12)$ & - \\
\hline $\mathrm{CH}_{3} \mathrm{CN}$ & $2.23(14)$ & $1.34(14)$ & $1.45(14)$ & $1.00(13)$ \\
\hline $\mathrm{CH}_{3} \mathrm{OH}$ & $1.08(13)$ & $2.95(12)$ & $2.79(12)$ & $2.00(13)$ \\
\hline $\mathrm{CH}_{3} \mathrm{CO}$ & $5.73(12)$ & $1.32(12)$ & - & - \\
\hline
\end{tabular}

the illumination from a nearby $\mathrm{OB}$ association. In this scenario we have assumed a geometry with a OB association illuminating the disk surface with a uniform enhanced UV field of $10^{4} G_{0}$. The physical properties of a disk around a low-mass star are very different, and the gravitational radius, $r_{\mathrm{g}}$, for an assumed $1 M_{\odot}$ star will be significantly smaller than that previously considered for a disk around a $10 M_{\odot}$ object. The predicted column densities for Models H-J are given in Table 6 for those species previously discussed in Table 4 .

Models $\mathrm{H}$ and I include a PDR, while model J excludes it. In model I, the region inside the PDR is warmer than in model H. None of these models includes an enhanced ionization rate. The general conclusions for Table 4 also apply here. It is remarkable that the averaged molecular column densities predicted for these species in this system are generally comparable to those in the disk around a massive star. The implication of Table 6 is, therefore, that the detections made for GL 2591, might be repeated for disks around low-mass stars illuminated by an OB association, for example in Orion.

We complete this discussion by showing in Table 7 a list of species predicted to be abundant, and potentially detectable in disks around low-mass stars illuminated by a nearby OB association (Models $\mathrm{H}$ and I). In general, as indicated in Table 5, averaged column densities predicted for models $\mathrm{H}$ and I are comparable to those for models A, $\mathrm{B}$ and C. However, there are several exceptions; for example $\mathrm{HCO}^{+}$is much less abundant, while $\mathrm{CH}_{3}, \mathrm{CH}_{2} \mathrm{CN}$, $\mathrm{SO}_{2}$ and $\mathrm{HCS}$ are much more abundant in the low-mass 
Table 6. Predicted Column Densities of Species in the PDR around a Solar Mass Stellar System. Notation $a(b) \equiv a \times 10^{b}$.

\begin{tabular}{|c|c|c|c|}
\hline \multirow[b]{2}{*}{ Species } & \multicolumn{3}{|c|}{$\begin{array}{c}\text { Column Densities }\left(\mathrm{cm}^{-2}\right) \\
\text { for Models }\end{array}$} \\
\hline & Model H & Model I & Model J \\
\hline $\mathrm{CO}$ & $1.54(20)$ & $1.73(20)$ & $1.39(19)$ \\
\hline $\mathrm{HCN}$ & $5.67(16)$ & $5.45(16)$ & $2.03(15)$ \\
\hline $\mathrm{C}_{2} \mathrm{H}_{2}$ & $3.59(14)$ & $2.21(15)$ & $4.46(11)$ \\
\hline $\mathrm{CH}_{4}$ & $1.32(18)$ & $1.34(18)$ & $4.07(14)$ \\
\hline $\mathrm{NH}_{3}$ & $1.49(16)$ & $2.10(16)$ & $8.20(13)$ \\
\hline CS & $1.67(13)$ & $7.47(14)$ & $4.05(14)$ \\
\hline $\mathrm{SiO}$ & $2.94(16)$ & $2.86(16)$ & $2.05(15)$ \\
\hline $\mathrm{H}_{2} \mathrm{O}$ & $3.36(19)$ & $3.34(19)$ & $1.17(16)$ \\
\hline
\end{tabular}

case. The carbon chain $\mathrm{HC}_{5} \mathrm{~N}$ and the radical $\mathrm{H}_{2} \mathrm{CN}$ are probably undetectable in the low-mass case. Nevertheless, it is clear that there is a wide range of molecules with potentially detectable abundances in disks around low-mass stars.

At present there are no known observational data for gas of a suitably high temperature to make an accurate comparison with our model results. It should be noted that there is a significant difference in the results between the $1 M_{\odot}$ and $10 M_{\odot}$ case for molecules CO and CS. This is due to the very different lower layer temperatures in the PDR envelope between the high mass and low mass regions. The cooler temperatures of the $1 M_{\odot}$ disk greatly enhances the formation of $\mathrm{CO}$ and vice versa for CS.

As with the high mass case, a stellar-disk region without an enhanced UV produces vastly differing model results. This can be seen when comparing the Model J results with the other two models (see Table 6).

\section{Conclusions}

Disks around massive YSOs, and disks around low-mass stars illuminated by nearby OB associations, are eroded by photo-evaporation. However, within a critical radius, the evaporating material is gravitationally bound and creates a PDR-like envelope to the disk. We have explored the chemistry in a disk/PDR system and have compared it to a similar system without a PDR.

For the case of a massive central star, we predict that the disk/PDR system generates many abundant molecular tracers. These are generally insensitive to the adopted ionization rate, but do depend on the temperature structure that is adopted within the disk/PDR. Results of models are compared with the rather limited observational data for one disk around a massive star, GL 2591. Although the models are not intended as a "fit" to the data, the agreement is reasonably satisfactory. The contribution of the PDR to the chemistry is clearly significant, and we predict several molecules to be detectable.

Disks around low-mass stars irradiated by nearby OB associations also generate molecular tracers in the
Table 7. Predicted Column Densities of Species, for the LowMass Case, which may be observable. The cut-off column density is $10^{12} \mathrm{~cm}^{-2}$. Notation $a(b) \equiv a \times 10^{b}$.

\begin{tabular}{|c|c|c|}
\hline \multirow[b]{2}{*}{ Species } & \multicolumn{2}{|c|}{$\begin{array}{c}\text { Column Densities }\left(\mathrm{cm}^{-2}\right) \\
\text { for Models }\end{array}$} \\
\hline & Model H & Model I \\
\hline $\mathrm{OH}$ & $4.14(16)$ & $4.14(16)$ \\
\hline $\mathrm{CH}$ & $5.26(13)$ & $5.29(13)$ \\
\hline $\mathrm{CH}_{3}$ & $7.52(16)$ & $7.52(16)$ \\
\hline $\mathrm{C}_{2}$ & $5.18(15)$ & $5.18(15)$ \\
\hline $\mathrm{C}_{2} \mathrm{H}$ & $1.82(15)$ & $1.82(15)$ \\
\hline $\mathrm{HCO}$ & $3.61(12)$ & $1.70(13)$ \\
\hline $\mathrm{HCO}^{+}$ & - & $1.04(12)$ \\
\hline $\mathrm{H}_{2} \mathrm{CO}$ & $6.53(14)$ & $5.13(14)$ \\
\hline $\mathrm{H}_{2} \mathrm{~S}$ & $1.12(13)$ & $1.11(13)$ \\
\hline OCS & $6.24(12)$ & - \\
\hline CS & $7.47(14)$ & $1.69(13)$ \\
\hline HCS & $1.13(16)$ & $1.13(16)$ \\
\hline $\mathrm{H}_{2} \mathrm{CS}$ & $9.63(12)$ & $7.68(12)$ \\
\hline $\mathrm{SO}$ & 7.43(13) & $5.52(14)$ \\
\hline $\mathrm{SO}_{2}$ & $8.67(16)$ & $8.89(16)$ \\
\hline $\mathrm{NH}$ & $4.81(12)$ & $4.87(12)$ \\
\hline $\mathrm{NH}_{2}$ & $7.74(12)$ & $7.84(12)$ \\
\hline $\mathrm{NH}_{3}$ & $2.10(16)$ & $1.49(16)$ \\
\hline $\mathrm{CN}$ & $1.01(13)$ & $1.01(13)$ \\
\hline $\mathrm{CH}_{2} \mathrm{CN}$ & $4.56(18)$ & $4.56(18)$ \\
\hline $\mathrm{HC}_{3} \mathrm{~N}$ & $1.48(13)$ & $2.10(12)$ \\
\hline $\mathrm{C}_{2} \mathrm{H}_{3}$ & $7.68(12)$ & $7.68(12)$ \\
\hline $\mathrm{C}_{2} \mathrm{H}_{4}$ & $7.29(13)$ & $7.24(13)$ \\
\hline $\mathrm{C}_{3}$ & $7.15(12)$ & $7.15(12)$ \\
\hline $\mathrm{HNC}$ & $2.02(16)$ & $2.11(16)$ \\
\hline $\mathrm{C}_{3} \mathrm{H}_{4}$ & $3.13(12)$ & $2.26(12)$ \\
\hline $\mathrm{CH}_{3} \mathrm{CN}$ & $3.37(13)$ & $3.00(13)$ \\
\hline $\mathrm{CH}_{3} \mathrm{OH}$ & $1.70(13)$ & $5.36(12)$ \\
\hline $\mathrm{CH}_{3} \mathrm{CO}$ & $2.71(13)$ & $2.90(12)$ \\
\hline
\end{tabular}

disk/PDR system that are distinct from the system without a PDR. There have been no reported detections, to date, of any of the species predicted; however their averaged column densities are generally similar to those predicted (and, in four cases, observed) for a disk around a massive star. Detection of these species in a disk around a low-mass star would identify that disk erosion was occurring and therefore that the disk may be undergoing planet formation.

Acknowledgements. The authors wish to thank Yuri Aikawa and Gerd-Jan van Zadelhoff for private communications. S. Viti and D. A. Williams acknowledge the financial support of PPARC for their research. We are grateful to a referee for comments that improved an earlier version of this paper. 


\section{References}

Aikawa, Y., \& Herbst, E. 1999, A\&A, 351, 233

Aikawa, Y., Umebayashi, T., Nakano, T., \& Miyama, S. 1997, ApJ, 486, L51

Aikawa, Y., Umebayashi, T., Nakano, T., \& Miyama, S. 1998, in Chemistry and Physics of Molecules and Grains in Space, of Faraday Discussions, 109, 281

Bally, J., Sutherland, R. S., Devine, D., \& Johnstone, D. 1998, ApJ, 116, 293

Boonman, A. M. S., van Dishoeck, E. F., Lahuis, F., Wright, C. M., \& Doty, S. D. 2000, ESASP, 456, 67

Carr, J. S., Evans, N. J., Lacy, J. H., \& Zhou, S. 1995, ApJ, 450, 667

Charnley, S. B., \& Tielens, A. G. G. M. 1992, in Astrochemistry of Cosmic Phenomena, IAU Symp., 150, 317

Chiang, E. I., \& Goldreich, P. 1997, ApJ, 490, 368

Dutrey, A., Guilloteau, S., \& Guélin, M. 1997, A\&A, 317, L55

Girart, J. M., Rodriguez, L. F., Anglada, G., et al. 1994, ApJ, 435,145

Goldsmith, P. F., Langer, W. D., \& Velusamy, T. 1999, ApJ, 519, L173

Helmich, F. P., van Dishoeck, E. F., Black, J. H., et al. 1996, A\&A, 315, 173

Hollenbach, D., Johnstone, D., Lizano, S., \& Shu, F. 1994, ApJ, 428,654

Igea, J., \& Glassgold, A. E. 1999, ApJ, 518, 848

Johnstone, D. 2000, in Astrochemistry: From Molecular Clouds to Planetary systems, IAU Symp., 197, 403
Johnstone, D., Hollenbach, D., \& Bally, J. 1998, ApJ, 499, 758 Lada, C. J., Thronson, H. A., Smith Jr., H. A., Schwartz, P. R., \& Glaccum, W. 1984, ApJ, 286, 310

Lahuis, F., \& van Dishoeck, E. F. 2000, A\&A, 355, 699

Lepp, S., \& Dalgarno, A. 1996, A\&A, 306, L21

Liszt, H. S., \& Lucas, R. 2000, A\&A, 355, 333

Millar, T. J., Bennett, A., Rawlings, J. M. C., Brown, P. D., \& Charnley, S. B. 1991, A\&AS, 87, 585

Millar, T. J., Farquhar, P. R. A., \& Willacy, K. 1997, A\&AS, 121,139

Mitchell, G. F., Curry, C., Maillard, J.-P., \& Allen, M. 1989, ApJ, 341, 1020

O'Dell, C. R. 1998, ApJ, 115, 263

Ohishi, M., Irvine, W., \& Kaifu, N. 1992, in Astrochemistry of Cosmic Phenomena, IAU Symp., 150, 171

Ruffle, D. P., Hartquist, T. W., Rawlings, J. M. C., \& Williams, D. A. 1998, A\&A, 334, 678

Ruffle, D. P., \& Herbst, E. 2000, MNRAS, 319, 837

Scappini, F., Cecchi-Pestellini, C., Codella, C., \& Dalgarno, A. 2000, MNRAS, 317, 6

van der Tak, F. F. S., van Dishoeck, E. F., Evans, N. J., Bakker, E. J., \& Blake, G. A. 1999, ApJ, 522, 991

van Dishoeck, E. F., \& Black, J. H. 1988, ApJ, 334, 771

van Zadelhoff, G. J., van Dishoeck, E. F., Thi., W. F., \& Blake, G. A. 2001, A\&A, 377, 566

Viti, S., \& Williams, D. A. 1999a, MNRAS, 305, 755

Viti, S., \& Williams, D. A. 1999b, MNRAS, 310, 517

Wagenblast, R. 1992, MNRAS, 259, 155

Willacy, K., \& Langer, W. D. 2000, ApJ, 544, 903 